(London, North West) and local practitioners from outside England (USA, Canada, Brazil). Transcripts were analysed thematically.

Results

15 decision makers from planning, urban design, housing and leisure services participated across three workshops. Participants' conceptualisations of what constitutes evidence included routine data, maps, guidelines, anecdotes, case studies and academic research. Local evidence in the form of case studies was particularly valued. Evidence was seen as useful for rationalising policies, including providing justifications for funding. The politicised nature of local government can leave evidence deprioritised in the face of competing interests and political ideologies. The current economic environment in local government has resulted in reduced resources for gathering and analysing data and a lack of funds to commission research. Negative perceptions about the complexity and relevance of academic studies were also barriers to evidence utilisation.

Conclusion

This study highlights both the need for locally relevant evidence and some possible differences in evidence cultures between public health services and the local government built environment sector. By better understanding evidence cultures this study helps lay the foundation for more informed policy making in this new environment.

Key messages

- Locally relevant evidence on social determinants of health is most valuable to local government decision-makers, but there are constraints on using evidence in policy making in local government.

- The English public health reform is an opportunity to integrate the social determinants of health approach into local government policies, but requires understanding of the cultures of evidence.

\section{How evidence on the social determinants of health is understood and utilised by non-health sector decision makers in four countries: qualitative findings Elizabeth Tyner McGill}

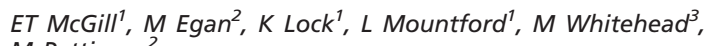
M Petticrew ${ }^{2}$

${ }^{1}$ Department of Health Services Research and Policy, School for Public Health Research, London School of Hygiene and Tropical Medicine, London, UK

${ }^{2}$ Department of Social and Environmental Health Research, School for Public Health Research, London School of Hygiene and Tropical Medicine, London, UK

${ }^{3}$ Department of Public Health and Policy, School for Public Health Research, LiLaC collaboration, Liverpool, UK

Contact: elizabeth.mcgill@lshtm.ac.uk

Background

In 2012, a major reform in England included the transfer of responsibility for delivery of local public health services from the National Health Service to local government. The move presents an opportunity to more fully incorporate an evidenceinformed social determinants of health approach into the policies of local government sectors associated with the 'upstream determinants' of health. Public health and local government, however, may conceive of, and utilise, evidence differently. Integrating evidence-informed decisions across public health and local government requires an understanding of what types of evidence are relevant to local government decision makers. Using qualitative methods, we explored how local government practitioners outside the health sector understand and use evidence, and barriers to its use.

\section{Methods}

Focus group workshops were held with decision makers working in the built environment in two English regions 